\title{
Una nuova lettura di Matteo Maria Boiardo (a proposito di un bel libro recente)
}

\author{
Edoardo Fumagalli
}

Fra i contributi recenti dedicati a Matteo Maria Boiardo, un posio di grande rilievo occupa il libro di Cristina Zampese, "Or si fa rossa or pallida la luna". La cultura classica nell' "Orlando Innamorato", pubblicato nel dicembre 1994 a Lucca da Maria Pacini Fazzi, come volume 13 della collana "L'Unicorno". È un saggio così denso, vario e nuovo, da fornire a qualunque lettore una massa notevolissima di informazioni, che vanno ben al di là del pur vasto campo di interessi indicato dal sottotitolo, e giungono anzi a offrire gli elementi che consentono di ribaltare i giudizi tradizionali sul conte di Scandiano e sulla sua opera. Proseguendo e approfondendo le indicazioni di alcuni degli studiosi più avvertiti, che negli ultimi decenni hanno scavato nella cultura del Boiardo, cogliendone alcuni riflessi nel poema, Cristina Zampese ha ricostruito con pazienza e grande perspicacia un quadro che, se avrà bisogno qua e là di essere ritoccato o addirittura corretto, appare nella sostanza assolutamente persuasivo, in forza del numero e della qualità degli elementi radunati e interpretati.

Affrontare il problema della cultura classica nell'Orlando Innamorato mette subito dinanzi a una situazione che può sconfortare o stimolare, a seconda del temperamento di chi si accinge all'impresa: una situazione di difficoltà provocata dall'assenza, allo stato attuale delle ricerche, della biblioteca del poeta. Sappiamo, naturalmente, che egli conobbe gli autori normali della scuola, ovvi per qualunque persona colta, da Virgilio a Lucano, da Stazio a Ovidio, da Seneca tragico a Orazio: per non nominare che i principali fra i poeti; e sappiamo che egli addirittura volgarizzò alcuni prosatori, quali Erodoto, Senofonte, Apuleio, Cornelio Nepote, ricorrendo per i greci a una traduzione latina; ma non possiamo, proprio a causa della perdita o del non riconoscimento dei suoi libri, seguire la fase preparatoria, di studio e di meditazione degli autori, che normalmente veniva affidata alle postille scritte nei margini. $\mathrm{Ci}$ mancano, insomma, gli elementi indispensabili per penetrare nell'officina del Boiardo, e siamo costretti a indagare, per dire così, soltanto sul prodotto finito, per riconoscere nelle opere, e soprattutto nell'Innamorato, quelle presenze dei classici che consentano di valutare i modi attraverso i quali gli autori sono stati assimilati e utilizzati.

Come è noto, e come Cristina Zampese opportunamente richiama all'inizio del suo libro (19-24), si conoscono a tutt'oggi due soli manoscritti di autori classici 
appartenuti ai Boiardo: il Seneca, Tragoediae di Londra, British Library, Add. 11.986 e il Lucano, British Library 11.990, scritto a Ferrara da "Iacobus Iuliani de Portiolo" nel marzo del 1378: si tratta di due testi fondamentali, come l'indagine rivela, ma non c'è alcuna prova che quei due codici siano appartenuti a Matteo Maria, ${ }^{1}$ probabilmente quest'ultimo avrà posseduto una parte almeno dei libri del nonno Feltrino e del padre, ma non si va oltre la verosimiglianza e, in ogni caso, quei libri, se pure sopravvivono, non sono stati riconosciuti. ${ }^{2}$ In queste condizioni, l'autrice enuncia nelle pagine iniziali un programma minimo, volto allo studio di alcune opere sicuramente presenti al Boiardo: quelle ch'egli stesso ha volgarizzato; quelle presenti nella biblioteca ducale di Ferrara; quelle non ignorabili, perché costituivano il patrimonio normale di chiunque avesse seguito un dignitoso corso di studi. Si tratta, da parte di Cristina Zampese, di un punto di partenza: che il libro, con la ricchezza delle sue acquisizioni, supera nel giro di poche pagine, in forza di un'analisi che è di solito del tutto persuasiva, e che, anche quando non convince fino in fondo o addirittura induce al dissenso, obbliga tuttavia a un proficuo supplemento di indagine. Prima di discutere alcuni dei temi trattati, non sarà fuori luogo descrivere brevemente il volume.

Dopo la Premessa e l'Introduzione, comincia la trattazione vera e propria, suddivisa in tre parti di ampiezza disuguale: La formazione classicistica del Boiardo, che costituisce una sorta di antefatto propedeutico all'analisi, occupa le pagine 9-48, mentre le due sezioni principali, L'appropriazione dei classici e Gli autori "praestantiores familiaresque magis", si stendono rispettivamente da $\mathrm{p}$. 49 a p. 190 e da p. 191 a p. 262; seguono la Cor.clusione (263-69), le indicazioni bibliografiche e cinque indici: "dei luoghi dell'Orlando innamorato citati nel testo", "dei luoghi di altre opere boiardesche citati nel testo", "dei luoghi classici citati nel testo", "dei nomi, dei luoghi e delle cose notevoli", "dei personaggi, dei luoghi e delle cose notevoli del poema citati nel testo".

Qui intendo soffermarmi sulle sezioni seconda e terza, anche per discutere alcune delle conclusioni; ed è ovvio cominciare dalla parte prima e più cospicua, quella dedicata all'appropriazione dei classici nel poema. Conviene subito avvertire che il lavoro di Cristina Zampese non è, neppure in queste pagine, un mero elenco di "fonti" intese in senso positivistico e ordinate per autori; viene largito piuttosto un esame ragionato di alcuni degli episodi del racconto, che consentono di riconoscere quali sono stati i testi messi a frutto dal Boiardo, e di irradiare le acquisizioni anche su altre parti dell'Innamorato. I titoli stessi dei capitoli di questa sezione sono di per sé parlanti: I grandi episodi fantastici (II corno magico; Falerina; Morgana; Le armi di Ettore; La riviera del Riso), Orchi e spelonche, ovvero il gusto dell'orrido (Rocca crudele; Il libro "che avea di sangue tutta la scrittura"; L'orco di Lucina), Le "novelle" (Tisbina; Leodilla; Doristella); Echi formali (L'architettura della trama narrativa; I latinismi linguistici; Figure 
retoriche; Strutture sintattico-ritmiche). I "latinismi linguistici" possono servire come introduzione a una materia che, pur nella sua varietà, presenta una coerenza che fin qui era in gran parte sfuggita; la ricerca mette in luce, infatti, un elemento importante: mentre negli Amorum libri Pier Vincenzo Mengaldo aveva a suo tempo rilevato la presenza di spunti lessicali, più che tematici, che consentivano di cogliere la presenza di un classico, nel poema Boiardo mostra di essere sollecitato dai temi, così che Cristina Zampese può con piena ragione dichiarare che "l'impiego di latinismi nell'Orlando innamorato quasi mai costituisce una spia per l'individuazione di una fonte" (163), illustrando poi in modo convincente le rare eccezioni; è, questa, una conclusione che deve essere pienamente condivisa, soprattutto da parte di chi abbia una qualche dimestichezza con i volgarizzamenti boiardeschi: non è possibile qui esemplificare, ma è fuor di dubbio che, quando utilizza anche nel poema un episodio o un passo che aveva già tradotto in volgare, il Boiardo di norma riduce i latinismi, spesso violenti nei volgarizzamenti, alla forma consueta all'uso della lingua cancelleresca della seconda metà del Quattrocento. ${ }^{3} \mathrm{Ma}$ il diverso comportamento del Boiardo, per quanto riguarda i debiti contratti con i classici, nel canzoniere e nel poema, anticipa di oltre un secolo quanto Giambattista Marino avrebbe sostenuto nella lettera famosa a Claudio Achillini, là dove leggiamo che l'imitazione "nella invenzione e nelle cose" pertiene al poema eroico, mentre quella "nella sentenza e nelle parole" è più tipica del genere lirico; e tuttavia, se ci si limitasse a queste considerazioni generali, si farebbe torto grave non solo a Boiardo, ma anche alla sua interprete, che ha il merito di sottolineare come il poeta si muova con una libertà che gli permette di utilizzare, a proposito dello stesso tema o delle stesse situazioni, spunti e suggerimenti che gli arrivano da opere diverse: a volte anche molto diverse; si trovano così coinvolti, come fonti della stessa avventura, e mescolati insieme in una sintesi che ha del prodigioso, autori classici e medievali, con una forte presenza, naturalmente, dei romanzi francesi.

Mi limito a un piccolo esempio, tratto dalle pp. 53-64 che analizzano l'episodio del corno magico. Molti studiosi hanno insistito sulla derivazione dell'avventura di Orlando in 1.24 da Ovidio, Metamorfosi 3 e 7, cioè dalle imprese di Cadmo e di Giasone; il quadro si fa molto più complesso sotto la lente di Cristina Zampese, che mostra come il Boiardo tenesse presente anche Valerio Flacco, Stazio, Plinio e addirittura la Historia destructionis Troiae di Guido delle Colonne. Infatti i versi dell'ott. 52

e via tagliò la testa sanguinosa; quella poi prese il conte, e remirando ben gli parve quel capo orribil cosa, ch'era vermiglio, d'oro e verde e bruno; fuor di quel trasse e denti ad uno ad uno 
trovano il loro riscontro più persuasivo nelle parole "caput eius a collo trucidanti mucrone divisit. A cuius faucibus evulsis dentibus ipsos continuo per sulcos factos inseruit in arato campo dudum a bobus" dello scrittore duecentesco (5960); ma subito riprendono le fonti classiche, abilmente fuse e spiritosamente occultate con il rinvio a Turpino, come per esempio in 53-54:

Turpin, che mai non mente in alcun loco, dice che penne uscirno a poco a poco.

Penne depinte, dico, de cimieri uscirno a poco a poco de la terra, e dapoi gli elmi e' petti de' guerrieri e tutto il busto integro si disserra. Prima pedoni, e poscia cavallieri uscir, tutti cridando: - Guerra, guerra! con trombe e con bandiere, a gran tempesta: ciascun la lancia verso Orlando arresta.

I primi versi riecheggiano il noto Ovidio, Met. 3.107-10 ("primaque de sulcis acies apparuit hastae, / tegmina mox capitum picto nutantia cono, / mox umeri pectusque onerataque bracchia telis / existunt crescitque seges clipeata virorum"), così come gli ultimi si rifanno a Met. 7.131-32 ("praeacutae cuspidis hastas / in caput Haemonii iuvenis torquere parantes"); ma quelli centrali dipendono dalle Argonautiche di Valerio Flacco, 6.610-11 ("Martius hic primum ter vomere fusus ab ipso / clangor et ex omni sonuerunt cornua sulco") e soprattutto 628-29 ("sic undique densant / terrigenae iam signa duces clamore tubaeque"), ${ }^{4}$ mentre i versi 56,7-8 ("contra alla gente che gli ariva intorno, / che, pur mo' nata, die' morir quel giorno") imitano Stazio, Tebaide 4.555-56 ("Terrigenae comites illos, gens Martia, cingunt, / quis aevi mensura dies"). E non è ancora tutto qui, perché, come la studiosa opportunamente annota, il commento staziano di Lattanzio Placido pare, in questa come anche in altre occasioni, avere indirizzato la fantasia del Boiardo, con l'annotazione "Mensura dies". Quibus nascendi moriendique dies" (59-61); se poi, come mi sembra legittimo, si aggiunge al quadro il dantesco "verdi come fogliette pur mo nate" di Purg. 8.28, il risultato è un mosaico straordinariamente vivace, che pienamente giustifica la reazione contro "il diffuso pregiudizio sul carattere irriflesso e non colto del poema” (167 n. 334).

Mi sono ristretto, com'è naturale, a un caso limitato, che mostra però l'ampiezza e la profondità dell'indagine, e la ricchezza dei risultati. Non sarà più possibile, dopo questo volume, considerare il Boiardo alla stregua di uno dei numerosi canterini della sua generazione, magari più abile degli altri e sostenuto da una nativa eleganza che lo salva anche nei momenti più stanchi, e in ogni caso significativo soprattutto in quanto precursore dell'Ariosto; ed è un peccato che Riccardo Bruscagli per la sua bella edizione commentata dell'Innamorato 
(Torino, Einaudi, 1995) sia potuto giungere, nelle more della stampa, solo a citare in bibliografia questo libro, senza avere la possibilità di travasarne nelle note le conquiste.

Dopo l'ampia sezione dell"'appropriazione dei classici" segue, come si diceva, quella dedicata agli "autori praestantiores familiaresque magis", che già nella formulazione, derivante dalla Politia litteraria di Angelo Decembrio, tende a immettere il Boiardo nella tradizione culturale ferrarese di derivazione guariniana. I capitoli sono dedicati rispettivamente a Stazio, a Lucano ("e a Seneca tragico", come recita il titolo), a Valerio Flacco, a Virgilio, e Ovidio; il sesto e ultimo, "Boiardo lettore raffinato. Saggi di lettura di due autori meno diffusi", sul quale si tomerà brevemente, riprende e conclude la trattazione di Claudiano e introduce i riscontri con il Cupido cruciatus di Ausonio.

Anche qui si dovrà esemplificare castamente; ma, prima di procedere nell'analisi e nella presentazione del volume, è opportuno manifestare uno dei rari punti di dissenso. Cristina Zampese accenna diverse volte non solo alla possibilità, ma, se interpreto bene, addirittura alla probabilità che il Boiardo abbia letto e utilizzato autori greci in lingua originale; e non si tratta solo di autori, quali Erodoto od Omero, dei quali erano disponibili traduzioni latine che avrebbero potuto facilitare il compito (non si deve peraltro dimenticare che proprio la versione del Valla costituiva il filtro per il volgarizzamento della vasta opera storica), ma addirittura di Apollonio Rodio, cioè di un autore che, per usare le parole di Gianvito Resta citate anche dalla studiosa, "non ha suscitato l'entusiasmo e la curiosità erudita delle prime generazioni umanistiche, ma è stato una tarda e faticosa 'scoperta' della più agguerrita cultura degli ultimi decenni del Quattrocento" (229 n. 101). ${ }^{5}$ Sarebbe ingiusto attribuire alla studiosa, a questo proposito, una sicurezza ch'ella di solito ovatta con formule quali "non senza esitazione" (229) o "eventuale conoscenza" (230); ma non si può negare che, esclusa per motivi diversi la mediazione della traduzione di Bartolomeo Fonzio o dell'imitazione di Basinio da Parma (230), i numerosi accostamenti, da lei operati, delle ottave boiardesche agli esametri di Apollonio tendono a stabilire un legame che non mi pare possa reggere alla verifica. Tutti i passi che vengono accostati, infatti, non sembrano andare oltre una generica somiglianza, derivante dall'identità della materia; e non c'è dubbio che su questo terreno, che coinvolgerebbe non solo la conoscenza pressoché perfetta del greco da parte del Boiardo, ma anche la lettura e l'assimilazione di testi assolutamente periferici, le prove devono essere solidissime. Converrà, credo, fino a un'altamente improbabile dimostrazione in contrario, seguitare a vedere in Boiardo non un filologo desideroso di esplorare campi poco frequentati, ma un poeta dotato delle normali conoscenze delle persone colte del suo tempo: con l'aggiunta, che 
decisamente lo stacca da quella compagnia, di una mirabile capacità combinatoria che il libro di Cristina Zampese per la prima volta rivela in tutta la sua estensione.

Questo non contrasta con la presenza, sopra accennata, di Claudiano, sfruttato dal poeta fin dai componimenti giovanili, e neppure di Ausonio: il quale da tempo era ben noto, anche se non era un autore propriamente della scuola, ed aveva avuto gli onori della stampa fin dal 1472, subito dopo la stagione delle grandi edizioni dei classici curate a Roma dall'Aleriense. Con tutto questo la loro efficacia sulle ottave dell'Innamorato, esibita, per quanto riguarda Claudiano, in diverse parti del volume, e per entrambi in modo particolare alle pp. 259-62, non cessa di essere molto significativa; anche se, dallo studio della Zampese, risulta che gli autori più sfruttati sono, comprensibilmente, altri, e in modo particolare, oltre Virgilio e Ovidio e Stazio, i due oltranzisti Lucano e Seneca tragico. Su di essi, e in particolare sul primo, è opportuno soffermarsi, anche perché il comportamento del Boiardo può far nascere altre prospettive di ricerca.

$$
\text { * * * }
$$

A Lucano e a Seneca tragico sono dedicate specificamente le pp. 217-25, ma i loro nomi e i rinvii alle loro opere sono continui lungo tutto il volume. Se si facesse il conto delle citazioni, probabilmente Lucano prevarrebbe anche su Virgilio e su Ovidio; ma ciò che più interessa sottolineare è che il poeta della Farsaglia svetta sugli altri non solo per il numero delle riprese individuate, ma anche per le loro caratteristiche. Basterebbe, a questo proposito, rilevare che il titolo stesso del libro di Cristina Zampese, Or si fa rossa or pallida la luna, riproduce, sì, uno splendido verso boiardesco, Inn. 2.6.7, che però a sua volta deriva da Phars. 5.546-50: "lunaque non gracili surrexit lucida cornu / aut orbis medii puros exesa recessus, / nec duxit recto tenuata cacumina cornu / ventorumque nota rubuit; tum lurida pallens / ora tulit voltu sub nubem tristis ituro". Proprio questo episodio presenta caratteri tali, da permettere di spingersi un po' innanzi su strade già peraltro accennate dalla studiosa.

Siamo all'inizio del canto sesto del secondo libro dell'Innamorato, quando, per usare il sommario allestito da Bruscagli nella sua edizione, "Rodamonte in Algeri, impaziente di prendere il largo, sfida i venti sfavorevoli ... e nonostante il parere avverso dell'esperto Scombrano decide di mettersi per mare" (2.628); si tratta, dunque, di una situazione già in partenza del tutto simile a quella che ha per protagonista, nel libro quinto del Bellum civile, il pescatore Amiclate di fronte a Cesare: e alla somiglianza delle vicende si accordano le riprese anche testuali, che qui metto in evidenza riproducendo i medesimi corsivi che Cristina Zampese introduce a p. 219.

Rispose a lui Scombrano: - Alto segnore, alla partita abbiam contrario vento; il mare è grosso e vien sempre maggiore. 
Ma io prendo de altri segni più spavento, ché il sol callando perse il suo vigore, $e$ dentro a i novaloni ha il lume spento; or si fa rossa or pallida la luna, che senza dubbio è segno di fortuna.

La fulicetta, che nel mar non resta, ma sopra al sciutto gioca ne l'arena, e le gavine che ho sopra alla testa, e quello alto aeron che io vedo apena, mi dànno annunzio certo di tempesta; ma più il delfin, che tanto se dimena, di qua di là saltando in ogni lato, dice che il mare al fondo è conturbato [2.6.7-8]

Tum pauper Amyclas:

"Multa quidem prohibent nocturno credere ponto; nam sol non rutilas deduxit in aequora nubes concordesque tulit radios: Noton altera Phoebi, altera pars Borean diducta luce vocabat. Orbe quoque exhaustus medio languensque recessit spectantes oculos infirmo lumine passus. Lunaque non gracili surrexit lucida cornu aut orbis medii puros exesa recessus, nec duxit recto tenuata cacumina cornu ventorumque nota rubuit; tum lurida pallens ora tulit voltu sub nubem tristis ituro.

Sed mihi nec motus nemorum nec litoris ictus nec placet incertus qui provocat aequora delphin, aut siccum quod mergus amat, quodque ausa volare ardea sublimis pinnae confisa natanti, quodque caput spargens undis, velut occupet imbrem, instabili gressu metitur littora cornix".

[Phars. 5.539-56]

L'accostamento è, come si vede, ferreamente persuasivo. Anzi, credo che si possa perfino andare oltre, anche sulla scorta di un suggerimento di Ettore Paratore, il quale, ripreso da Cristina Zampese, citava a proposito della "fulicetta" boiardesca Virgilio, Georg. 1.360-364 (Paratore 349):

Iam sibi tum curvis male temperat unda carinis, cum medio celeres revolant ex aequore mergi clamoremque ferunt ad litora, cumque marinae in sicco ludunt fulicae, notasque paludes 
deserit atque altam supra volat ardea nubem.

"La fulicetta, che ... sopra al sciutto gioca" deriva senza dubbio da questi versi; ma altrettanto fuori discussione è che sulla rielaborazione boiardesca abbiano influito anche quelli che, riferiti ad altro uccello, si incontrano poco più avanti, Georg. 1.388-89:

Tum cornix plena pluviam vocat improba voce et sola in sicca secum spatiatur harena.

Saremmo, fin qui, press'a poco nella norma della memoria associativa cui il libro di Cristina Zampese ci ha abituato; ma l'ampiezza stessa della ripresa da Lucano rende già a prima vista plausibile l'ipotesi che il Boiardo non si affidasse unicamente alle virtù mnemoniche, che pure dovevano essere cospicue e certo inimmaginabili per noi oggi, ma che avesse sotto gli occhi il testo lucaneo. Il sospetto viene confermato dalla circostanza che, accanto alle espressioni direttamente carpite alla Farsaglia, altre ve ne sono, che certamente derivano da uno dei prodotti tipici della scuola umanistica del secondo '400: il commento di Ognibene da Lonigo. 6

Già il v. 541 di Lucano viene spiegato nel modo seguente:

Nam sol non ru.] quia occidente sole si aer rubuerit significat diem crastinum serenum fore, sed hoc signum tunc non fuerat...,

dove "occidente sole" certo meglio corrisponde al boiardesco "il sol calando", e dove "signum" è solo una delle numerose occorrenze di questo vocabolo, assente dai versi della Farsaglia, ma presente due volte nell'ott. 7 di Boiardo (vv. 4 e 8) e a più riprese nel commento di Ognibene; tralasciando casi più minuti, decisiva è la spiegazione dei vv. 539-40 e 551:

Tunc pauper Amyclas] dicit Amyclas se multa signa vidisse, quae tempestatem significarent...

Sed mihi] sunt alia signa tempestatem significantia...,

alla quale corrisponde, dopo l'accenno di 74 , il verso finale dell'ottava:

che senza dubbio è segno di fortuna.

Si può anche osservare che all'aggettivo "pallida" di 77 corrisponde al v. 549 "lurida pallens", mentre la chiosa umanistica introduce proprio la forma adottata da Boiardo:

lurida] supra modum pallida.

sub nubem] nam ille pallor lunae significabat se paulo post sub nubem obscuratum iri, 
dove si può nutrire il sospetto non infondato che l'ultima espressione citata abbia contribuito alla nascita di 76 :

e dentro a i novaloni ha il lume spento.

Più significativo è però a questo punto leggere la chiosa al v. 556, dove Lucano introduce la "cornix" che lega questo passo alle Georgiche; e una volta di più ci viene in soccorso Ognibene da Lonigo:

instabili gressu] quia per arenam graditur. Vir.: Et sola in sicca secum spatiatur arena.

Vediamo allora che il commento è attivo su due fronti: da un lato fornisce, attraverso la perifrasi o la chiosa puntuale, l'interpretazione di un passo o il lessico per riprodurlo con variazioni; dall'altro, accostando luoghi paralleli, consente a chi se ne serve di accennare, senza alcuna fatica, ad altri autori, come in questo caso Virgilio.

La conseguenza è che, se pure Matteo Maria ebbe in casa il Lucano ora londinese e posseduto alla fine del secolo XIV dalla famiglia Boiardo, certamente utilizzò anche un incunabolo della Farsaglia con il commento di Ognibene da Lonigo.

Il libro di cui stiamo discorrendo rinnova radicalmente l'immagine tradizionale del poeta di Scandiano, presentandocelo nella prospettiva, mai tanto nitida, di un autore non solo dotto, ma anche capace di fondere in una sintesi abilissima fonti disparate, così da occultare, nella maggior parte dei casi, le giunture. Ma il pubblico cui Boiardo si rivolgeva era in grado di comprendere le finezze di un procedimento combinatorio tanto dissimulato, da rischiare di passare inosservato? In un suo articolo, che opportunamente Cristina Zampese ricorda a p. 8, Giorgio Pasquali si era posto da par suo il medesimo problema, e da par suo aveva risposto, osservando fra l'altro):

io sono convinto che le Georgiche non furono mai lette da un solo contadino, e del resto poco profitto ne avrebbero tratto gli agricoltori, perché l'intento didascalico, altrimenti che per Esiodo, è per Virgilio puro pretesto; tanto che determinazioni necessarie per l'uso pratico sono taciute ogniqualvolta avrebbero offuscato o appesantito la rappresentazione, quindi quasi sempre. (277)

La situazione per Boiardo è un po' diversa: non si trattava di leggerne l'opera per imparare i segreti della coltivazione e i contadini erano fuori scena; ma resta il problema di comprendere quali potessero essere le reazioni del pubblico di fronte ai mosaici di allusioni presente nell'Innamorato. 
La strada più ovvia e più semplice è offerta dai rifacitori; in particolare, il Rifacimento del Berni offre qualche indicazione interessante, che ha bisogno di essere sviluppata e approfondita.

Ho appena segnalato come in Inn. 2.68 ("La fulicetta, che nel mar non resta / ma sopra al sciutto gioca ne l'arena") sia attivo anche il ricordo di Virgilio, Georg. 1.388-89 ("Tum cornix plena pluviam vocat improba voce / et sola in sicca secum spatiatur harena"); l'allusione nascosta viene compresa dal Berni, che infatti accentua la vicinanza a questo passo latino in 2.6 .9 :

La fulicetta nell'acqua non resta, ma passeggia all'asciutto in su la rena.

Non so dire se questo accada spesso; certo non sempre, dal momento che ci sono casi in cui il Rifacimento lascia cadere uno o più elementi presenti nel Boiardo per imitazione dei classici. Scelgo ancora uno dei passi già sopra esaminati: il verso "con trombe e con bandiere, a gran tempesta" di Inn.1.24.54, che come indicato da Cristina Zampese a p. 61, deriva da Valerio Flacco, Arg. 7.629 ("terrigenae iam signa duces, clamorque tubaeque": riproduco qui la lezione adottata dalla studiosa), ma il Berni, che presumibilmente non aveva afferrato l'allusione, tradì la cultura elegante del modello quando (1.24.58 8) variò in

con trombe $e$ corni, che fu bella festa.

Sarà agevole, sulla scorta del ricchissimo apparato di rinvii contenuto nel libro, verificare anche da questo lato il comportamento di Francesco Berni, così da approfondire un aspetto non trascurabile del travisamento, non solo linguistico, del poema.

Desidero soffermarmi su un ultimo aspetto del volume di Cristina Zampese: le innovazioni, non sempre chiare, introdotte dal Boiardo rispetto alle proprie fonti. L'episodio più caratteristico è quello orroroso di Stella e Marchino, dove tra l'altro leggiamo (1.8.43):

La cruda Stella, menando gran festa, a Marchin va davanti in viso fello, e li appresenta l'una e l'altra testa de' figli, ch'io servai dentro a un piatello.

La tecnofagia ha, naturalmente, anche altri precedenti; qui tuttavia Boiardo utilizza senza dubbio un racconto di Erodoto 1.119 (Zampese 37-38), che tuttavia sia neli'originale, sia nella traduzione latina del Valla parla non di un "piatello" 
ma di un "cesto". Il particolare più interessante è che di un "piato", e non di un cesto, il Boiardo riferisce anche nel suo volgarizzamento dello storico greco:

ad Arpago solamente forno date di quelle [maniere de vivande] che de la carne del figliolo erano composte, e ne la fine de la cena gli fece entro ad uno piato portare el capo e piedi e mano del fanciullo, che servate avea. ${ }^{7}$

Cristina Zampese, di fronte al comportamento del Boiardo, che "concorda con se stesso e si discosta inequivocabilmente sia dall'originale greco, sia dalla versione latina", dichiara di rinunciare "a indagarne oltre le ragioni", dopo aver brillantemente individuato la fonte dell'episodio (38); a me pare probabile che qui Boiardo, con un procedimento audace ma non eccezionale, contamini la storia di Astiage con quella di Giovanni Battista, così come viene raccontata nel cap. 14 di Matteo, dal quale cito, e dal cap. 6 di Luca:

Die autem natalis Herodis saltavit filia Herodiadis in medio et placuit Herodi. Unde cum iuramento pollicitus est ei dare quodcumque postulasset ab eo. At illa praemonita a matre sua, Da mihi, inquit, hic in disco caput Ioannis Baptistae. Et contristatus est rex; propter iuramentum autem et eos qui pariter recumbebant iussit dari misitque et decollavit Ioannem in carcere. Et allatum est caput eius in disco et datum est puellae et attulit matri suae.

Ma, naturalmente, accanto alle fonti dirette, evangeliche, occorre tener presente il numero grande di dipinti, che raffiguravano la testa del Battista posata su un "piato" o "piattello", come dice Boiardo, o su un tagliere, come scriveva per esempio Lucrezia Tornabuoni. ${ }^{8}$ La presenza di testi echi biblici nell'Innamorato è del resto ancora tutta da studiare, ma probabilmente un'indagine di questo tipo riserverebbe qualche sorpresa. Certamente si può portare l'esempio della disavventura capitata a Orlando in 1.29, quando "Origille infinocchia mirabilmente il Conte, e lo lascia a cercare, se vuole, la visione del paradiso e dell'inferno sulla tomba di Nino, mentre essa fugge via con Brigliadoro" (Rajna 204). Scrive Boiardo (53 5-8):

Quivi ritrova che sepolto è Nino, qual fu già re di questo tenitoro, e fece Ninivè, l'alta citate, che in ogni verso è lunga tre giornate;

le parole in corsivo si rifanno al libro di Giona, 3.3: "Et surrexit Ionas et abiit in Niniven iuxta verbum Domini, et Ninive erat civitas magna itinere trium dierum"; ma, ancora una volta, non basta. Già Giulio Razzoli (91-92) ha segnalato a suo tempo non solo, cio che appare piuttosto ovvio, che "il sepolcro di Nino è pure menzionato da Ovidio in Metam. (4.85) come il luogo al quale dovevano trovarsi insieme Piramo e Tisbe", ma anche i punti di contatto fra 
l'episodio e un'analoga vicenda del Guiron; si può aggiungere che altre letture ancora hanno presumibilmente offerto a Boiardo lo spunto per l'ottava 49:

E così cavalcando a passo a passo,
ragionando più cose intra di loro,
a mezo il prato ritrovarno un sasso,
che è scritto tutto intorno a littre d'oro,
e trenta gradi, dalla cima al basso,
avea tagliato con netto lavoro;
per questi gradi in cima se saliva
a quel petron, che asembra fiamma viva.

La descrizione del sepolcro di Nino, infatti, pare rifarsi al racconto di Diodoro, 2.7, che Niccolò Leoniceno, sempre ammirato e lodato dal Boiardo, aveva volgarizzato come segue (ed. Venezia: Giolito, 1542, 37v):

Nino, composto quel stato a proposito, con li thesori de Battri quasi innumerabili a Nina si ritornò, licentiati et premiati li socii, dove Semiramis li partorì Nino il secondo. Ma poco dipoi, venuto il primo Nino a morte, lassò il regno a la donna, che li fè un sepulchro nel palazzo alto nuove stadii (come dice Ethesia) et largo dieci, il quale, essendo la città in pianura, appariva da lunge molti stadii come una smisurata torre, che anchora si dice essere in piè, benché li Medi ruinassino Nina quando si fer signori de Assyria.

Una volta di più, se queste proposte possono essere accolte, trova conferma la insospettata ricchezza del poema. Altra questione, s'intende, è di accertare se il Boiardo abbia sempre dimostrato il polso fermo necessario per padroneggiare l'abbondanza straripante dei riferimenti e delle invenzioni: su questo piano, probabilmente, $i$ tradizionali giudizi limitativi saranno confermati anche da nuove indagini; è un aspetto, in ogni caso, che esula dagli intenti molto più modesti di questo articolo, che non si prefiggeva altro scopo che quello di presentare il libro di Cristina Zampese: un volume, occorre ripetere, bello e suscitatore di problemi. Se non si provasse un po' di ripugnanza nell'usare un aggettivo tante volte impiegato a sproposito, si potrebbe perfino dire: stimolante.

\section{Università di Friburgo}

\section{NOTE}

1 Si pud aggiungere che questo copista in quello stesso mese scrisse almeno un altro Lucano, il Breslau R 97 (Ziegler 64-66); scritto anch'esso a Ferrara ("Lucani liber explicit quem ego domnus Jacobus Juliani de Portiolo filius Ferarie scripsi. 1378 et de mense martij"), rimase presumibilmente in cittaे, se, come lo Ziegler riferisce, al f. 108v "diversae manus saec. XV./XVI. conscribillarunt totum; leguntur frustula ex Quintiliano et Sallustio sumpta, dicta Socratis, saepius quod est manubrium, nomen guarini..." (65). 
2 Per Feltrino si veda Mariotti; oltre che con Feltrino, Poggio Bracciolini ebbe rapporti non del tutto tranquilli, per quanto riguarda la restituzione di libri, anche con un figlio, che per questioni di date non può essere il padre di Matteo Maria: Bracciolini, 3.423-24. Un caso a parte è quello che conceme il De Architectura di Leon Battista Alberti, che in una sua lettera, ricordata dalla Zampese (23), Matteo Maria dichiara di avere letto al duca Ercole I d'Este; trattandosi di un libro a stampa, non poteva essere altro che un esemplare dell'edizione fiorentina del 1485: ma non pare che sia stata avviata un'indagine per accertare se quel volume sia giunto fino a noi

3 Mi permetto di rinviare al contributo, in corso di stampa, letto al Convegno "Il Boiardo e il mondo estense nel Quattrocento", 13-17 settembre 1994, dove ho cercato di mettere in risalto l'altemativa "acinace"- "scimitarra", "obsidione"-"assedio", rispettivamente nel volgarizzamento di Erodoto e nell'Innamorato.

4 Queste ultime parole sono variamente atteggiate nei manoscritti; qui ho riprodotto il testo secondo la forma più diffusa, ma è da osservare che Cristina Zampese ha "clamorque tubaeque".

5 La citazione è tratta da Resta 639, dove anche si offre la spiegazione del fenomeno: "non perché non fossero approdati da tempo in Italia alcuni importanti manoscritti, ma proprio per una diffusa carenza di interesse per quella difficile opera, che, pertanto, era poco ricercata; sostituita, come favola, nel favore dei lettori, con pigra considerazione, dal più accessibile Argonauticon di Valerio Flacco. Ne derivò con una ben scarsa circolazione di mss., una conoscenza limitata a pochi studiosi". Importanti anche i rilievi espressi in nota, e soprattutto l'osservazione che "solo a partire dal 1470 circa l'opera di Apollonio, studiata spesso come fonte dell'Argonauticon di Valerio Flacco, merito più attenta considerazione da parte della cultura umanistica".

6 Ricordo che importanti considerazioni sulla necessità di tener presenti, accanto alle opere dei classici, i loro commenti, sono svolte da Tissoni Benvenuti, in particolare pp. 296 ("mi sembrano di grande utilita i commenti noti a corte o nati per la corte: da essi infatti impariamo non solo quali testi allora circolavano, ma come venivano letti, quali significati si attribuivano loro e quali erano le pagine ritenute più interessanti") e seguenti.

7 Cito da una edizioncina dell'infanzia di Ciro, nel volgarizzamento boiardesco, che ho allestito per un'occasione famigliare. Aggiungo che, trattandosi di un fascicolo artigianale e fuori commercio, sarò ben lieto di inviarlo a chi, tramite la direzione di questa rivista, avesse la bontà di mostrarsene interessato.

8 Tomabuoni, ottave 137, 142, 144. "Tagliere" ha anche Filelfo 38.21 ("Io una gratia ti dimarido sola: / che I capo di Giovanni or di presente / in un taglier me die, e non sia fola") e 38 ("La testa gli taglio senza tenzone, / e messa in taglier in don la porta / a quella che di ciò parea cagione").

\section{OPERE CITATE}

Boiardo, Matteo Maria. Orlando innamorato. Ed. Riccardo Bruscagli. Voll. 2. Torino: Einaudi, 1975.

Bracciolini, Poggio. Lettere. Ed. Helène Harth. Voll. 3. Firenze: Olschki, 1984-87.

Philelpho, Francesco. La Vita dil sanctissimo lohanne Baptista. Milano: Biblioteca Trivulziana, ms. 732.

Mariotti, Italo. "Tre epigrammi per casa Boiardo". Miscellanea 2: 445-81.

Miscellanea Augusto Campana. Voll. 2. Padova: Antenore, 1981.

Paratore, Ettore. "L'Orlando innamorato" e l'Eneide". Il Boiardo e la critica contemporanea. Atti del Convegno di studi su Matteo Maria Boiardo, Scandiano - Reggio Emilia 25-27 aprile 1979. Ed. Giuseppe Anceschi. Firenze: Olschki, 1970.347-75.

Pasquali, Giorgio. “Arte allusiva”. Pagine stravaganti. 2. Firenze: Sansoni, 1968. 275-82. 
Rajna, Pio. Le fonti dell'Orlando Furioso. Ristampa della seconda edizione 1900 accresciuta d'inediti. Ed. Francesco Mazzoni. Firenze: Sansoni, 1975.

Razzoli, Giulio. Per le fonti dell' Orlando innamorato di M. M. Boiardo. Milano: Albrighi, Segati e C., 1901.

Resta, Gianvito. "Vegio, Basinio e l' Argonautica di Apollonio Rodio. Miscellanea 2: 639-69.

Tissoni Benvenuti, Antonia. "Note preliminari al commento dell'Inamoramento de Orlando". II commento ai testi. Atti del Seminario di Ascona, 2-9 ottobre 1989. Ed. Ottavio Besomi e Carlo Caruso. Basel - Boston - Berlin: Birkhäuser Verlag, 1992. 277-307.

Tomabuoni, Lucrezia. "Vita di sancto Giovanni Baptista". Fulvio Pezzarossa, I poemetti sacri di Lucrezia Tornabuoni. Firenze: Olschki, 1978.

Ziegler, Konrat. Catalogus codicum Latinorum qui in bibliotheca urbica Wratislaviensi adservantur. 1915. Hildesheim - New York: Georg Olms Verlag, 1975. 